\title{
Influence of the Wall on the Dynamic
} Behavior of Homogeneous Tubular Reactors with a Highly Exothermic Reaction

\section{GERHART EIGENBERGER ${ }^{1}$}

Institut fuer Systemdynamik und Regelungstechnik, Universitaet Stuttgart, West Germany

The influence of the tube wall on the behavior of tubular reactors with a highly exothermic reaction was studied. The effect of heat accumulation in the wall has a considerable influence upon the transient behavior of the reactor. Because of this influence excess temperatures can occur if the feed temperature is lowered or the flow velocity is raised. For a gas phase reaction the transient behavior is determined almost completely by the heat capacity of the wall. An example of multiple steady states caused by axial heat conduction in the wall is given, and the influence of heat conduction in the wall is compared with that in the reaction mixture.

Tomogeneous tubular reactors are usually described in terms of a plug flow 1 or diffusion model. The temperature of the reactor wall is assumed constant with time of operation. However, only the coolant temperature can be held constant while the wall temperature will be between that of the coolant and the reaction mixture. Thus, the wall temperature is influenced by changes in the reaction temperature, and the wall temperature in turn influences the behavior of the reaction. This wall influence is studied below. A highly exothermic first-order reaction ( $\mathrm{A} \rightarrow$ products) is considered. The simple reaction mechanism does not restrict the general validity of the results since the important dynamic effects are the result of exothermic acceleration of the reaction.

\section{Tubular Reactor with Liquid Phase Reaction}

Homogeneous liquid phase reactions in tubular reactors are often described by the plug flow model, Equations 1-3:

$$
\begin{gathered}
\frac{\partial c}{\partial t}+v \frac{\partial c}{\partial z}=-r_{(\mathrm{c} . \mathrm{T})} \\
\frac{\partial T}{\partial t}+v \frac{\partial T}{\partial z}=\frac{2 \alpha_{1}}{r_{1 P} c_{p}}(T=-T)+\frac{\left(-\Delta H_{R}\right)}{p c_{p}} r_{(\mathrm{e} . \mathrm{T})} \\
\text { with } r_{(\mathrm{e} . \mathrm{T})}=k_{\mathrm{o}} c e^{-\mathrm{B} / R T}
\end{gathered}
$$

'Present address: Department of Chemical Engineering, Northwestern University, Evanston, Ill. 60201 . 
Table I. Constants of the Liquid Phase Reaction

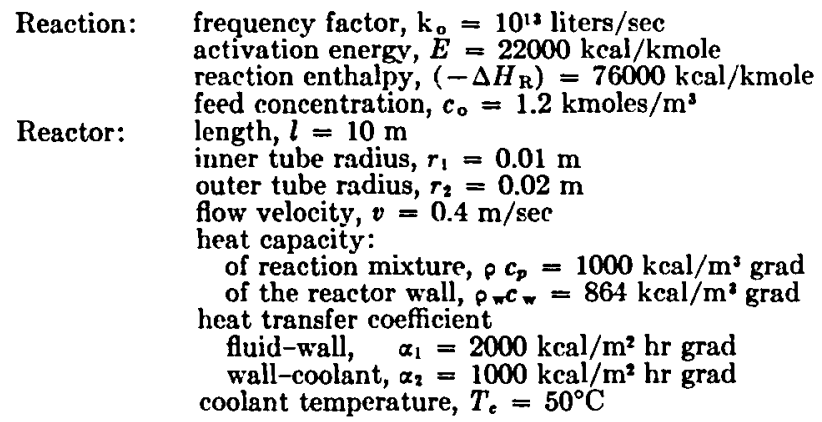

To account for the wall influence, an energy balance for the wall must be added. Consider the radial mean temperature of the wall. If the axial heat conduction in the wall is neglected, the wall has only a cumulative effect, and its energy balance is:

$$
\frac{\partial T_{w}}{\partial t}=\frac{2 \alpha_{1} r_{1}}{\rho_{*} c_{w}\left(r_{2}^{2}-r_{1}^{2}\right)}\left(T-T_{w}\right)+\frac{2 \alpha_{2} r_{2}}{\rho_{w} c_{w}\left(r_{2}^{2}-r_{1}^{2}\right)}\left(T_{e}-T_{w}\right)
$$

with initial and boundary conditions:

$$
\begin{gathered}
c(0, z)=c_{0}(z) ; T(0, z)=T_{0}(\mathrm{z}) ; T_{*}(0, z)=T_{\mathrm{mi1}}(z) \\
c(t, 0)=c_{0} ; T(t, 0)=T_{0}
\end{gathered}
$$

The values of the constants in Equation $1-4$ are given in Table 1 .

Figure 1 shows the transient of the system caused by a step increase in feed temperature (time $t$ as parameter). The front of the disturbance moves with flow velocity $v$ through the reactor. For a plug flow reactor without wall influence, temperature and concentration would jump from their old steady-state value to the new one, and the transient would be finished within one residence time. However, since the heat transfer to the wall is limited, establishment of the new steady state is delayed. Here the transient takes more than two residence times.

A step decrease of feed temperature shows an unexpected result. As shown in Figure 2, a decrease in feed temperature temporarily increases the maximum temperature. Similar behavior is observed if the flow velocity is suddenly increased (Figure 3). The reverse behavior occurs if the flow velocity is lowered (Figure 4). This phenomenon is caused by the heat capacity of the wall. If the temperature maximum is moved downstream by a sudden change of operating conditions, the wall will first retain its high temperature where the former maximum was located (see e.g., Figure $3, t=5 \mathrm{sec}$ ). To remove this surplus heat, heat fluxes from the wall to the reaction mixture. If the temperature maximum moves upstream, the wall at the front of the reactor must be heated. The required amount of heat will be taken from the reaction mixture so that the maximum temperature during this transient is lower than its steady-state value.

This phenomenon can be observed experimentally (1). The upper part of Figure 5 shows measured temperatures at different locations in a $10-\mathrm{m}$ long tubular reactor. Temperature profiles at discrete times are shown in the lower section. The reaction is the homogeneous liquid phase oxidation of ethanol with the overall stoichiometry: 

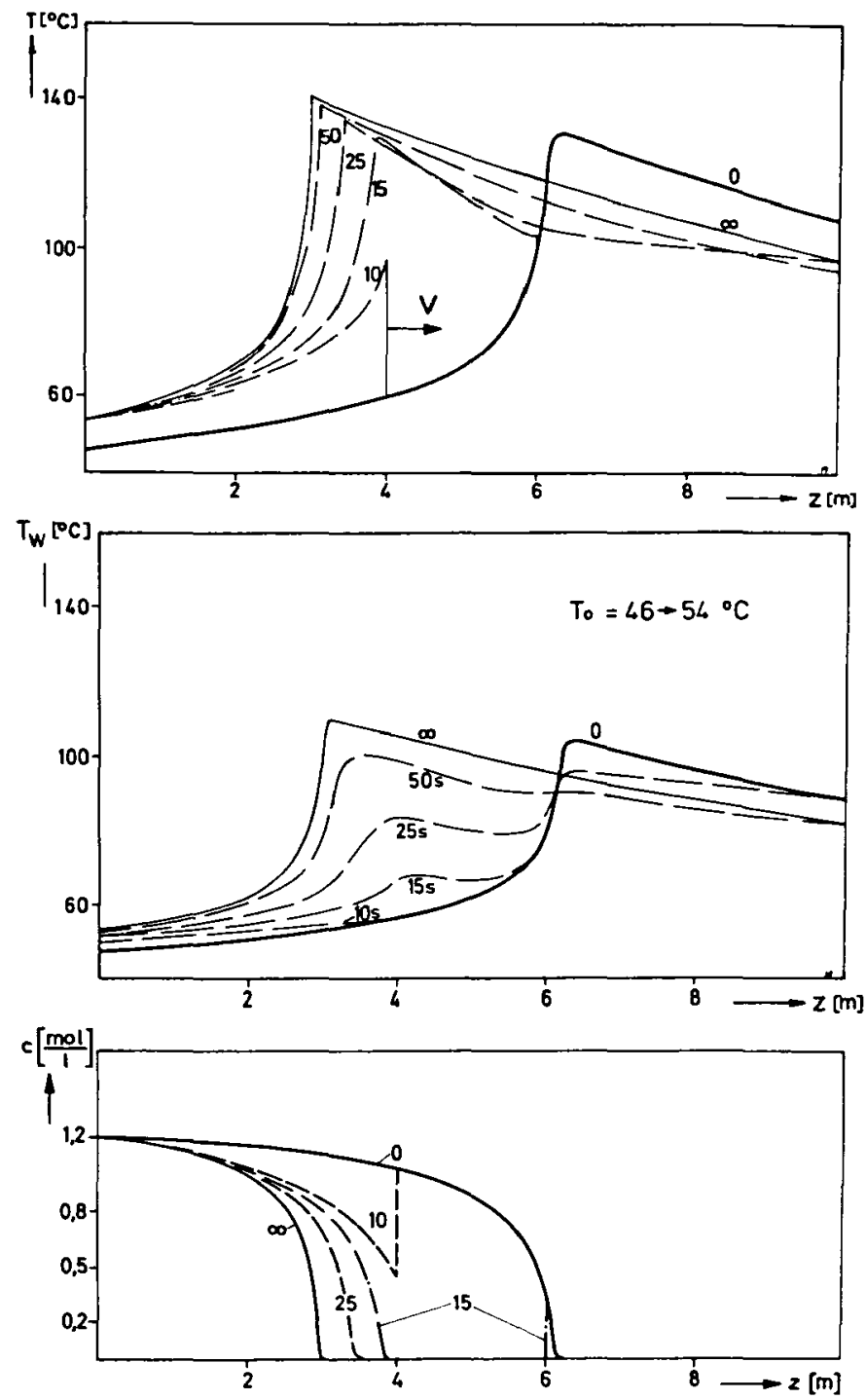

Figure 1. Transient profiles of the liquid phase reactor upon a step increase in feed temperature

$$
\mathrm{C}_{2} \mathrm{H}_{5} \mathrm{OH}+2 \mathrm{H}_{2} \mathrm{O}_{2} \stackrel{\mathrm{Fe}(\mathrm{III})}{\longrightarrow} \mathrm{CH}_{3} \mathrm{COOH}+3 \mathrm{H}_{2} \mathrm{O}
$$

Figure 5 shows the transient temperatures measured when flow velocity was doubled (flow velocity was increased rampwise over $8 \mathrm{sec}$ ).

This effect has a counterpart in the behavior of creeping profiles in fixed bed reactors. Wicke et al. $(2,3,4)$ showed that the maximum temperature of 
a creeping profile lies beyond or below its steady-state value, depending on the direction of the creeping velocity. The maximum temperature varies for reasons similar to those above, and the fixed bed can provide an even higher heat capacity than the reactor wall.

So far the effect of axial heat conduction in the reactor wall has been neglected. This is permissible since the convective heat flow is much higher
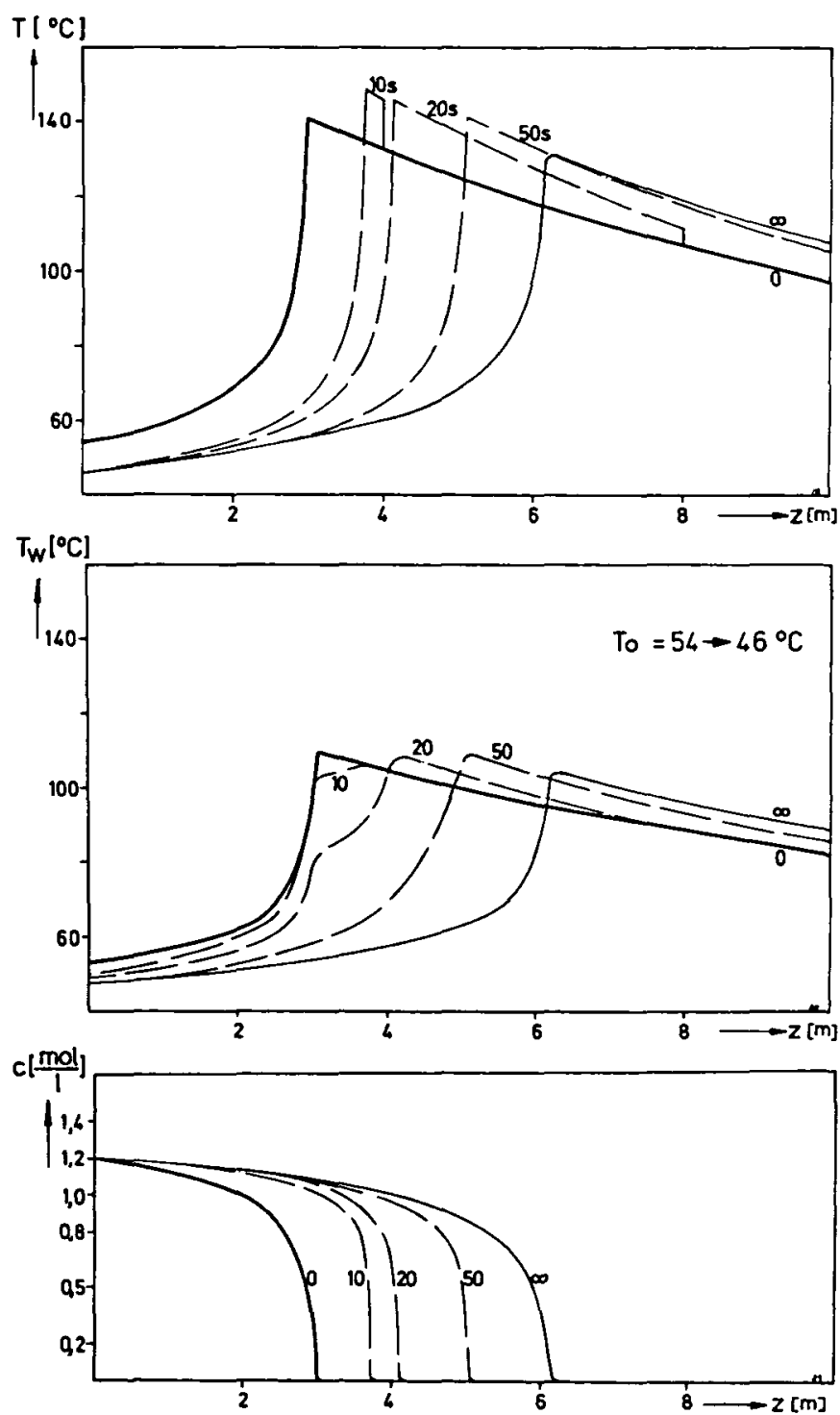

Figure 2. Transient profiles of the liquid phase reactor upon a step decrease in feed temperature 


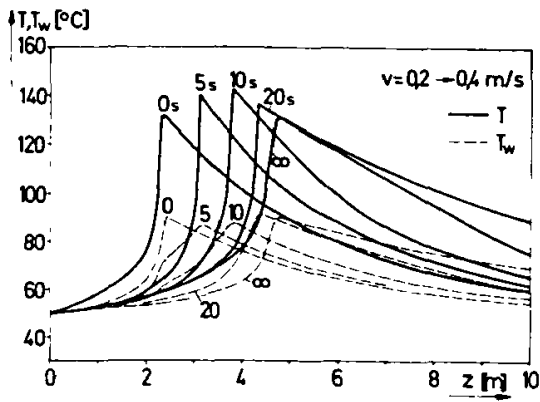

Figure 3. Transient profiles of the liquid phase reactor upon a step increase in the flow velocity

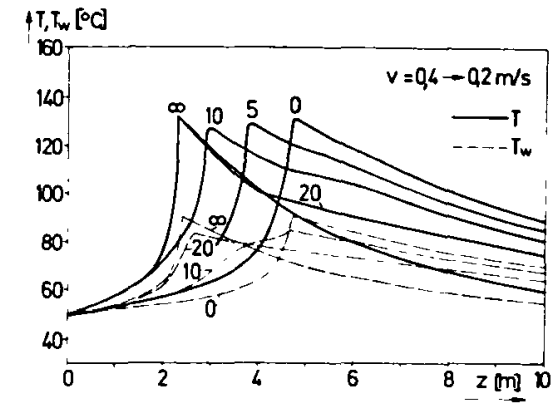

Figure 4. Transient profiles of the liquid phase reactor upon a step decrease in the flow velocity

than the heat conduction in the wall because of the high heat capacity of the liquid reaction mixture. For a gaseous reaction mixture this is not necessarily the case.

\section{Tubular Reactor with Gas Phase Reaction}

Because a gaseous reaction mixture is compressible, it is useful to consider a mass-dependent concentration measure. Using the weight fraction $g$ instead of the molar concentration $c$, Equations 1-3 yield:

$$
\begin{gathered}
\frac{\partial g}{\partial t}+v \frac{\partial g}{\partial z}=-\tau^{*}(\mathrm{~g}, \mathrm{~T}) \\
\frac{\partial T}{\partial t}+v \frac{\partial T}{\partial z}=\frac{\alpha_{1}}{\rho c_{\mathrm{p}}} \frac{2}{r_{1}}(T=-T)+\frac{\left(-\Delta H_{\mathrm{R}}\right)}{M \Gamma_{\mathrm{A}} c_{p}} r^{*}(0, T) \\
r^{*}(G, T)=k_{\infty} g_{\mathrm{A}} e^{-E / R T}
\end{gathered}
$$

Now the influence of axial heat conduction in the reactor wall will be included in the wall heat balance. Therefore Equation 4 must be extended by a heat conduction term. 


$$
\begin{gathered}
\frac{\partial T_{w}}{\partial t}=\frac{2 \alpha_{1} r_{1}}{\rho_{\pi} c_{\pi}\left(r_{2}^{2}-r_{1}^{2}\right)}\left(T-T_{w}\right)+\frac{2 \alpha_{2} r_{2}}{\rho_{w} C_{w}\left(r_{2}^{2}-r_{1}^{2}\right)}\left(T_{c}-T_{w}\right)+ \\
\frac{\lambda_{w}}{\rho_{w} C_{w}} \frac{\partial^{2} T_{w}}{\partial z^{2}}
\end{gathered}
$$

with new boundary conditions:

$$
g(0, t)=g_{0} ; T(0, t)=T_{0} ;\left.\frac{\partial T_{w}}{\partial z}\right|_{(0, t)}=\left.\frac{\partial T_{w}}{\partial z}\right|_{(l, t)}=0
$$

If one assumes constant pressure over the length of the reactor, only the following continuity equation must be added to the above model:

$$
\frac{\partial \rho}{\partial t}=-\frac{\partial(\rho v)}{\partial z}=\left\{\rho \frac{\partial v}{\partial z}+v \frac{\partial p}{\partial z}\right\}
$$


Figure 5. Measured transient temperatures of a laboratory reactor after doubling the flow velocity. Upper part: measured temperatures at different axial positions; lower part: transient profiles.

It is further assumed that the density rapidly establishes its quasi-steadystate value. With $\partial \rho / \partial t=0$, Equation 11 can be integrated, leading to the criterion for constant mass flux:

$$
p(z, t) v_{(2, t)}=p_{0} v_{0}=\text { constant }
$$

These assumptions are justified later.

If the reaction: $\mathrm{A} \rightarrow n \mathrm{~B}$, takes place in a mixture of ideal gases, density can be derived by: 


$$
\rho(z, t)=\rho_{\circ} \frac{T_{\circ}}{T_{(z, t)}} \frac{M_{\mathrm{A}}}{M_{\mathrm{\circ}}} \frac{1}{\left\{g(z, t)+n\left(g_{\mathrm{O}}-g(z, t)\right)+\frac{M_{\mathrm{A}}}{M_{\mathrm{o}}}-g_{\mathrm{O}}\right\}}
$$

$\rho$ and $v$ are now functions of $T(z, t)$ and $g(z, t)$, and the coefficients of the model equations are no longer constants. The behavior of the above model is discussed below. Values for the parameters are in Table II.

\section{Table II. Constants for the Gas Phase Reaction}

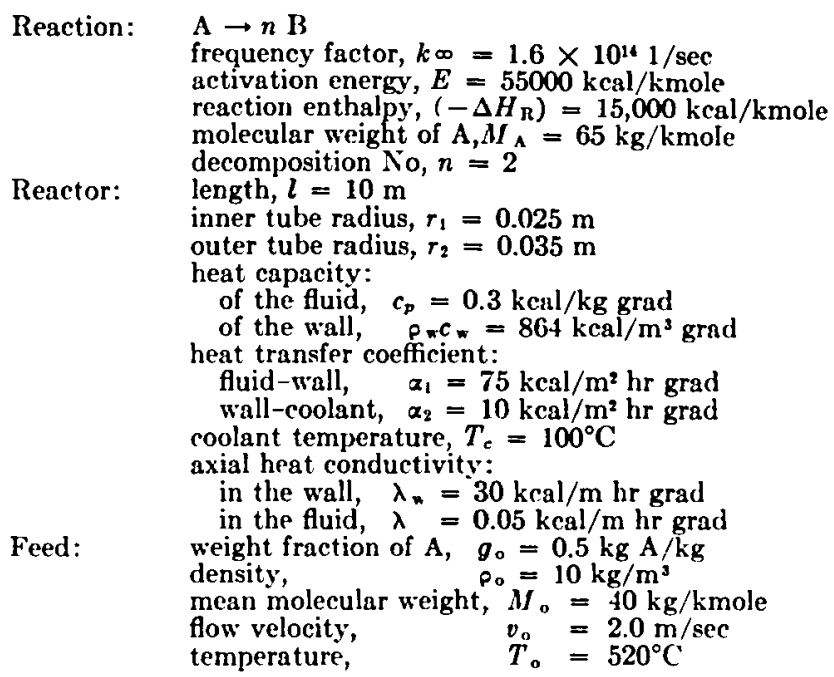

Figure 6 shows the steady-state profiles of the adiabatic reactor for the complete model (Equations 6-10,12,13) and for a reduced model $(\rho(z)=$ $\left.\rho_{0}, v(z)=v_{0}, \lambda_{w}=0\right)$. The differences are not large. Figures 7 and 8 show the dynamics of the reactor as feed temperature and flow velocity change. The effect of excess maximum temperatures, caused by feed temperature decrease or flow velocity increase, is even more pronounced than for a liquid reaction, and the transient lasts longer. It consists of two periods. During the first which takes one residence time the disturbance spreads over the whole reactor length. Because of the high heat capacity of the wall ( $v s$. the reaction mixture) the wall temperature does not change. The second period lasts several orders of magnitude longer. It is determined by the change in wall temperature while all the other variables are in quasi-steady state. This fact justifies the assumptions of quasi-steady density and constant pressure. Schuchmann (5) showed that dynamic changes in density and pressure in a gas phase reactor usually fade within fractions of the residence time, so they can influence only the short, first period. However, under certain conditions self-excited pressure and density oscillations can occur, causing the reactor to behave as an acoustic generator (6). These effects are not covered in our model.

\section{Influence of Axial Heat Conduction}

Comparisons of the calculations with and without a heat conduction term show that the influence of axial heat conduction is not very significant in the above examples $(v \geq 1 \mathrm{~m} / \mathrm{sec})$. However, axial heat conduction provides a 
feedback mechanism which can cause instabilities and multiple steady states. This problem, first considered by van Heerden (7) has often been discussed, but the discussions have always been based on the diffusion model (without wall influence). Figure 9 shows that multiple steady states can also arise because of the back conduction of heat in the reactor wall. Note the temperature profiles of an upper and a lower stable steady state. The unstable state between the two stable states has not been calculated. Compared with the above examples the flow velocity in the area of multiple steady states was much smaller, and even with $v=0.1 \mathrm{~m} / \mathrm{sec}$ the hysteresis in feed temperature between ignition and extinction was only a few tenths of a ${ }^{\circ} \mathrm{C}$.
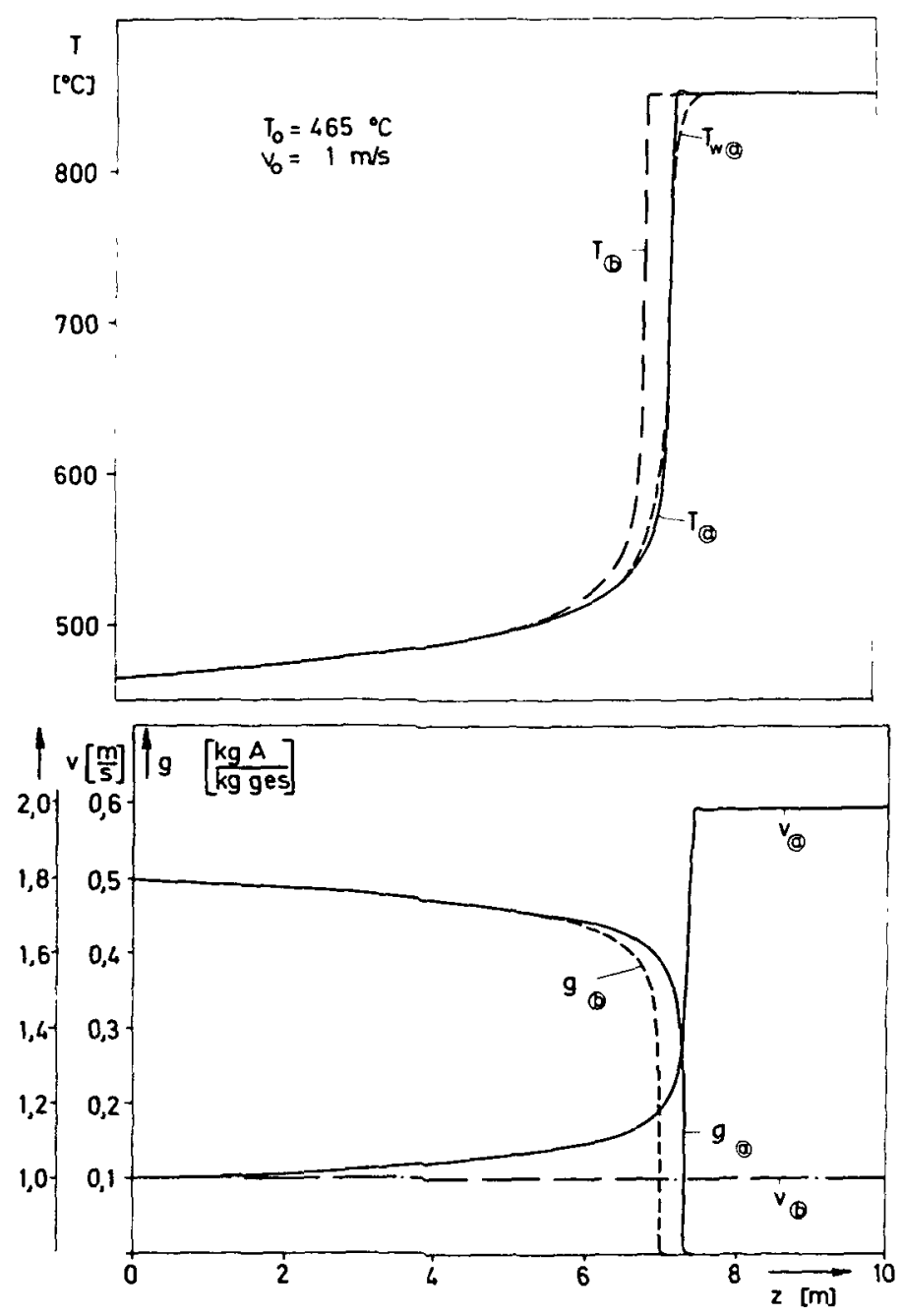

Figure 6. Steady-state profiles of the adiabatic gas phase reactor. (a) Complete model, $(b)$ reduced $\operatorname{model}\left(\rho(z)=\rho_{0}, \mathrm{v}(\mathrm{z})=\mathrm{v}_{0,}, \lambda_{x}=0\right)$. 

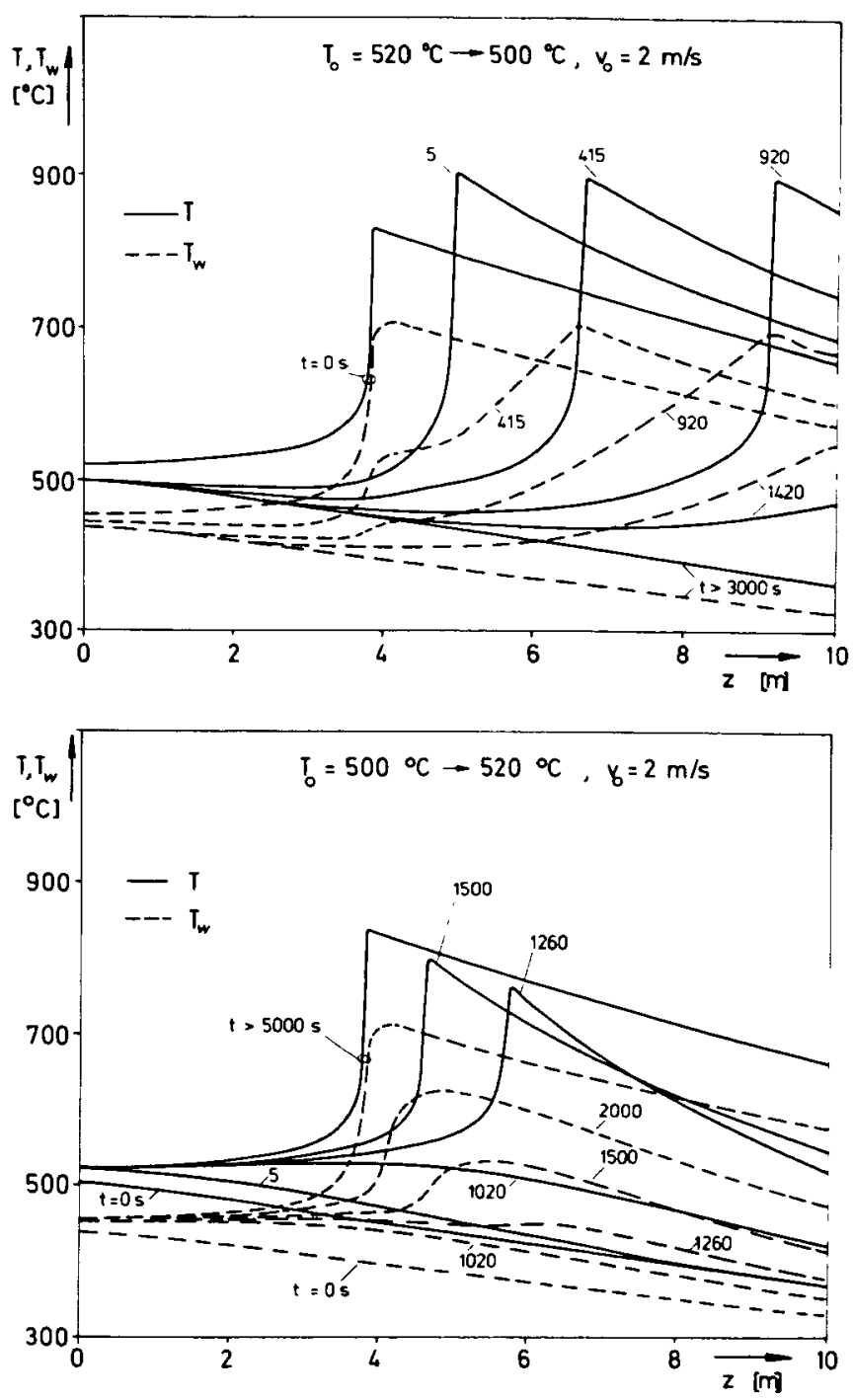

Figure 7. Transient profiles of the non-adiabatic gas phase reactor upon step changes in feed temperature

The distinct temperature peak in the ignited profile 2 is caused by the back conduction of heat into the main reaction zone. Thus, in addition to the heat of reaction released the fluid is heated by conductive heat backflow. In Figures 6 and 8 the peak is much smaller becauses of the larger influence of convective heat transport. (A similar temperature peak can also occur in calculating the diffusion model if $D_{\text {eff }}<\lambda_{\text {eff }} / \rho c_{p}$.)

To decide whether the influence of the back conduction of heat in reactor wall or mixture is the major cause of multiple steady states, the following estimate is given. Only an adiabatic reactor is considered. The backward 
conducted heat $Q$ in the lower steady state is decisive for the reaction ignition (and thus for the occurrence of multiple steady states). One portion of $Q$ is the heat conducted back in the wall:

$$
Q_{w}=\left(r_{2}^{2}-r_{1}^{2}\right) \times \lambda_{w} \frac{d T_{w}}{d z}
$$

Since the wall is isolated adiabatically and the wall temperature in the front of the reactor is almost equal to the fluid temperature (Figure 9), all $Q_{w}$ is transferred to the reaction mixture. The second portion of $Q$ is the heat conducted back in the reaction mixture:

$$
Q_{\mathrm{R}}=r_{1}^{2} \approx \lambda_{\text {off }} \frac{d T}{d z}
$$


Figure 8. Transient profiles of adiabatic gas phase reactor upon step changes in flow velocity

Profile $I$ in Figure 9 shows a steady state immediately before reaction ignition. $T_{w}$ and $T$ coincide almost over the whole length of the reactor. $A s$ an approximation assume $d T_{w} / d z \approx d T / d z$. Then only the ratio $Q_{\mathrm{w}} / Q_{\mathrm{R}}=\left(r_{2}^{2}-r_{1}^{2}\right) \lambda_{\mathrm{w}} /$ $r_{1}{ }^{2} \lambda_{\text {eff }}$ has to be considered to decide whether the influence of the wall heat conduction or the influence of the heat conduction in the reaction mixture is 
A very large stepwise decrease in feed temperature or increase in flow velocity leads to an immediate extinction of the reaction (e.g., in Figure 8 the flow velocity has to be raised above $15 \mathrm{~m} / \mathrm{sec}$ ). However, the danger of additional excess temperatures still exists since large changes in operating conditions usually cannot be realized stepwise.

The influence of axial heat conduction is small and is negligible in liquid phase reactions. In gas phase reactions backward heat conduction in the wall can lead to multiple steady states if the flow rate is very low. The amount of backward conducted heat in the wall can be considerably higher than the backHow of heat from conduction in the reaction mixture.

\section{Nomenclature}

c molar concentration of reacting component $A$

$c_{p} \quad$ specific heat of reaction mixture

$c_{w} \quad$ specific heat of reactor wall

g mass fraction of reacting component $A$

$\hat{k}_{\mathrm{o}} \quad$ frequency factor of liquid phase reaction

$k_{\infty} \quad$ frequency factor of gas phase reaction

$l$ reactor length

$n$ decomposition number $(\mathrm{A} \rightarrow n \mathrm{~B})$

$r$ reaction rate of liquid phase reaction, $\mathrm{kmoles} / \mathrm{m}^{3} / \mathrm{sec}$

$r^{*} \quad$ reaction rate of gas phase reaction, $1 / \mathrm{sec}$

$r_{1} \quad$ inner tube radius

$r$.. outer tube radius

$t \quad$ time coordinate

$v$ flow velocity

$z \quad$ space coordinate

$E$ activation energy

$M_{A} \quad$ molecular weight of component A

$M_{0}$ mean molecular weight of feed

$R$ gas constant

$T$ reaction temperature

$T_{w} \quad$ wall temperature

$T_{\mathrm{c}} \quad$ coolant temperature

$\alpha_{1} \quad$ heat transfer coefficient fluid-wall

$\alpha_{2} \quad$ heat transfer coefficient coolant-wall

$\Delta H_{\mathrm{R}}$ reaction enthalpy

$\lambda$ axial heat conductivity in the fluid

$\lambda_{w} \quad$ axial heat conductivity in the wall

$\rho$ density of reaction mixture

$\rho_{w} \quad$ density of wall

\section{Subscripts}

s steady state

o reactor inlet

\section{Literature Cited}

1. Widmaier, H., unpublished data.

2. Wicke, E., Vortmeyer, D., Ber. Bunsenges. (1959) 63, 145-52.

3. Padberg, G., Wicke, E., Chem. Eng. Sci. (1967) 22, 1035-51.

4. Wicke, E., Padberg, G., Arens, H., Proc. European Symp. Chem. Reaction Eng., 4th, Brusselles, 1968, 425-37.

5. Schuchmann, H., Chem. Ing. Tech. (1969) 47, 868-77.

6. Gilles, E. D., Chem. Eng. Sci. (1967) 22, 745-758.

7. van Heerden, C., Chem. Eng. Sci. (1958) 8, 133-145.

Recerved January 2, 1974. 




Figure 9. Temperature profiles of an upper and a lower stable steady state caused by heat conduction in the wall

dominant. First, however, the concept of effective heat conduction in empty tubular reactors must be discussed. The effective heat conduction is the sum of the molecular heat conduction and the contributions from Taylor diffusion and eddy diffusion. The molecular heat conduction provides a feedback mechanism in a temperature ascent, but it is usually very small compared with other contributions. However, neither the contributions from Taylor diffusion nor those from eddy diffusion are real feedbacks since they account only for the spread in residence time distribution caused by non-plug flow. (There is no continuous backstream of fluid particles but only a retardation in forward movement.) Therefore, the values for effective heat conduction and effective diffusion should be reduced to those molecular effects if the diffusional model is used with a highly exothermic reaction in an empty tubular reactor. Otherwise the model will predict too high a backflow of heat.

Therefore in the ratio of the conductive heat flow in wall and fluid, only the molecular heat conductivity $\lambda$ is considered for the fluid. Using the values in Table II, the conductive heat flux in the wall exceeds the conductive heat flux in the fluid about 500 times. It can be assumed that in all cases where axial heat conduction can influence homogeneous tubular reactors, the amount of heat conduction in the wall may be significant.

\section{Conclusions}

Heat accumulation in the wall influences the dynamic behavior of a homogeneous tubular reactor with a highly exothermic reaction. The possibility of excess temperatures caused by decreased feed temperature or increased flow velocity can be important for operating and controlling such reactors. In case of an emergency shut off, a decrease of the feed temperature or an increase of the flow velocity to blow out the reaction should be avoided. An increase in the cooling rate and a decrease of feed concentrations seems is advised instead. 